\title{
Incremento del rendimiento y extracción nutrimental en jamaica mediante soluciones nutritivas
}

\section{Increased yield and nutrient extraction in roselle through nutrient solutions}

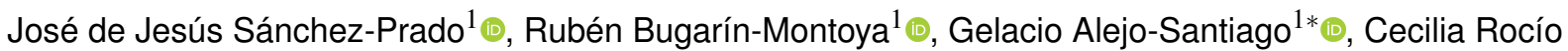 \\ Juárez-Rosete ${ }^{1}$ (D), Circe Aidín Aburto-González ${ }^{1}$ (D), Francisco Caro-Velarde ${ }^{1}$ (D) \\ ${ }^{1}$ Unidad Académica de Agricultura. Universidad Autónoma de Nayarit. Carretera Tepic-Compostela Km. 9.0 Xalisco. Nayarit. México. \\ *Autor de correspondencia: gelacioalejo@hotmail.com
}

Artículo científico recibido: 17 de mayo de 2018 aceptado: 01 de agosto de 2018

RESUMEN. La concentración de la solución nutritiva, es un factor importante que regula el crecimiento y desarrollo de las plantas en sistemas hidropónicos. El objetivo del trabajo fue identificar el mejor potencial osmótico de la solución nutritiva para incrementar el rendimiento de cálices de jamaica (Hibiscus sabdariffa L.) y cuantificar el requerimiento interno nutrimental (RI) de N, P, K, Ca y Mg. Se utilizó un diseño experimental completamente al azar con cuatro repeticiones. Los tratamientos fueron las concentraciones de $-0.018,-0.036,-0.054,-0.072,-0.090 \mathrm{MPa}$ de solución nutritiva de Steiner en potencial osmótico. La unidad experimental fue una maceta con una planta, empleando como sustrato escoria volcánica basáltica roja. Se realizaron siete muestreos destructivos durante el ciclo de cultivo. A la cosecha, se evaluó la producción de cáliz seco por planta y se eligió el tratamiento con mayor rendimiento para cuantificar los requerimientos nutrimentales del cultivo. El mejor potencial osmótico fue $-0.072 \mathrm{MPa}$ para la producción de cáliz seco, con rendimiento de $1.98 \mathrm{tha}^{-1}$. El RI de la biomasa total fue $2.86,0.50,2.80,1.71$ y $0.54 \%$ de N, P, K, Ca y Mg, respectivamente; lo que equivale en $\mathrm{kg} \mathrm{t}^{-1} \mathrm{de}$ cáliz seco a: N 93.1, P 16.4, K 91, Ca 55.7, Mg 17.7. El patrón de extracción nutrimental total en la biomasa aérea del cultivo fue en el orden $\mathrm{N}>\mathrm{K}>\mathrm{Ca}>\mathrm{Mg}>\mathrm{P}$. Las tasas de extracción nutrimental, variaron para cada nutrimento en las diferentes etapas fenológicas.

Palabras clave: Extracción nutrimental, Hibiscus sabdariffa L., materia seca, potencial osmótico, rendimiento de cáliz

ABSTRACT. The concentration of the nutrient solution is an important factor that regulates the growth and development of plants in hydroponic systems. The objective of this study was to identify the best osmotic potential of the nutrient solution to increase the yield of roselle (Hibiscus sabdariffa L.) calyces and quantify the internal nutritional requirement (IR) of N, P, K, $\mathrm{Ca}$ and $\mathrm{Mg}$. A completely randomized experimental design with four replicates was used. The treatments were the concentrations of $-0.018,-0.036,-0.054,-0.072$, and $-0.090 \mathrm{MPa}$ of Steiner's nutrient solution in osmotic potential. The experimental unit was a pot with a plant, using red basaltic volcanic slag as substrate. Seven destructive samplings were carried out during the crop cycle. At harvest, the dry calyx production per plant was evaluated and the treatment with the highest yield was chosen to quantify the nutrient requirements of the crop. The best osmotic potential was $-0.072 \mathrm{MPa}$ for dry calyx production, yielding $1.98 \mathrm{t} \mathrm{ha}^{-1}$. The IR of the total biomass was $2.86,0.50,2.80,1.71$ and $0.54 \%$ of $\mathrm{N}, \mathrm{P}, \mathrm{K}, \mathrm{Ca}$ and $\mathrm{Mg}$, respectively, which is equivalent in $\mathrm{kg} \mathrm{t}^{-1}$ of dry calyx to: $\mathrm{N} 93.1, \mathrm{P} 16.4, \mathrm{~K} 91, \mathrm{Ca} 55.7, \mathrm{Mg}$ 17.7. The pattern of total nutrient extraction in the aboveground biomass of the crop was in the order $\mathrm{N}>\mathrm{K}>\mathrm{Ca}>\mathrm{Mg}>\mathrm{P}$. Nutrient extraction rates varied for each nutrient in the different phenological stages.

Key words: Nutrient extraction, Hibiscus sabdariffa L., dry matter, osmotic potential, calyx yield.

\section{INTRODUCCIÓN}

La jamaica (Hibiscus sabdariffa L.) es un cultivo no tradicional en México y forma parte del sector de especias y plantas medicinales; a nivel mundial, ocupa el séptimo lugar como productor, pero se importa alrededor del $50 \%$ de la cantidad consumida (NICAEXPORT 2007). En México, entre los estados productores de jamaica, destacan Guerrero con $67 \%$ de la producción, Michoacán con el 12\%, Oaxaca 
con el $11 \%$, Puebla con el $6 \%$ y Nayarit con el $3 \%$, con rendimientos entre 260 y $650 \mathrm{~kg} \mathrm{ha}^{-1}$, con media nacional de $280 \mathrm{~kg} \mathrm{ha}^{-1}$ (SIAP 2017). Los bajos rendimientos se deben a la escasa tecnología que se emplea en los sistemas de producción (Caro-Velarde et al. 2012). Pero es posible alcanzar mayores rendimientos, como se ha demostrado al mejorar el manejo agronómico mediante fecha de siembra, espaciamiento entre plantas, densidad de población (EINaim 2012), lámina de riego apropiada (Babatunde y Mofoke 2006), materiales genéticos sobresalientes (Caro-Velarde et al. 2012, Ariza-Flores et al. 2014), y policultivos, entre otros aspectos (Ruíz-González y Victorino-Ramírez 2014).

La fertilización es un factor decisivo que impacta el rendimiento de cálices de jamaica, cuando el agua de riego no es un factor limitante (AboBaker y Mostafa 2011). Al respecto, investigaciones relacionadas con la fertilización del cultivo de jamaica, se enfocan en determinar la dosis de aplicación de gallinaza y nitrógeno (Ottai et al. 2006), fertilizantes compuestos (Egharevba y Law-Ogbomo 2007), biofertilizantes (Hassan 2009), nitrógeno en diferentes estaciones lluviosas (Giginyu y Fagbayide 2009), estiércol bovino y nitrógeno, y suministro de fertilizantes solos o en combinación con estiércol (Oyewole y Mera 2010). Pero se carece de información precisa de la demanda nutrimental del cultivo, que permita determinar la dosis de fertilización de acuerdo con las condiciones edafoclimáticas de las distintas regiones productoras del país (Dahmardeh 2012). La demanda nutrimental es la cantidad de nutrimento que requiere un cultivo para satisfacer las funciones metabólicas durante su ciclo de crecimiento y desarrollo (Etchevers 1999). Se calcula con base en la meta de rendimiento y el valor del requerimiento interno (RI) de un nutrimento en particular (Rodríguez et al. 2001), el cual corresponde a la concentración porcentual ideal del nutrimento en la biomasa aérea total en cosecha (Greenwood et al. 1980). El RI también se expresa en $\mathrm{kg}$ de nutrimento por tonelada de producto cosechado (Bugarín-Montoya et al. 2011) y su determinación se realiza con el establecimiento de ensayos de fertilización en campo o invernadero, con dosis crecientes de los nutrimentos de interés aplicadas al suelo, cuantificando el rendimiento de un cultivo en particular (Bugarín-Montoya et al. 2002). Un procedimiento alternativo es el empleo de los sistemas hidropónicos, donde el uso de una solución nutritiva mejora el control de la condición nutrimental, permitiendo manipular con mayor precisión que en suelo, las concentraciones nutrimentales y medir su efecto en el crecimiento y rendimiento de los cultivos (Vargas-Canales et al. 2014).

La concentración de la solución nutritiva en el ambiente radical, es un factor determinante que regula el crecimiento y desarrollo de los cultivos en sistemas hidropónicos (Moreno-Pérez et al. 2011). Su adecuado manejo durante las etapas fenológicas de un cultivo, la convierte en una herramienta técnica importante en la horticultura (Sonneveld y Voogt 2009). Debido a lo anterior, el objetivo fue determinar el mejor potencial osmótico de la solución nutritiva para incrementar el rendimiento de cálices de jamaica (Hibiscus sabdariffa L.) y cuantificar el requerimiento interno nutrimental de N, P, K, Ca y Mg.

\section{MATERIALES Y MÉTODOS}

El experimento se realizó en un invernadero de $8 \mathrm{~m}$ de ancho, $42 \mathrm{~m}$ de largo y $6.5 \mathrm{~m}$ de alto; con techo de dos aguas cubierto con polietileno, ventilación cenital y lateral protegida con malla antiáfidos, ubicado en la Universidad Autónoma de Nayarit, con coordenadas de $21^{\circ} 29^{\prime} 31^{\prime \prime} \mathrm{LN}, 104^{\circ} 53^{\prime} 31^{\prime \prime}$ LO, en la ciudad de Tepic, Nayarit a $952 \mathrm{msnm}$. Se empleó la variedad de jamaica UAN-6, obtenida en el programa de mejoramiento genético de la Unidad Académica de Agricultura. Se utilizaron plántulas obtenidas a partir de semilla en contenedores de poliestireno de 200 cavidades, previamente desinfectadas con una solución de hipoclorito de sodio (Cloralex ${ }^{\circledR}$ ) y se utilizó turba comercial Sunshine \# $3^{\circledR}$ como sustrato. El trasplante se realizó cuando las plantas tenían cuatro hojas verdaderas a los 40 días después de la siembra.

El experimento se estableció en un diseño completamente al azar, compuesto por cinco tratamientos y cuatro repeticiones. Los tratamientos 
fueron las cinco concentraciones iónicas de la solución nutritiva de Steiner (1961), expresadas en potencial osmótico $\left(\psi_{o}\right)$ : $-0.018,-0.036,-0.054,-0.072$, $-0.090 \mathrm{MPa}$ (Tabla 1). Durante el ciclo del cultivo se realizaron siete muestreos destructivos, en los dos primeros se aumentó el número de repeticiones a cinco plantas para asegurar la obtención de muestra para el análisis químico, en los siguientes cinco muestreos las repeticiones fueron de cuatro plantas, por lo que se usaron 150 plantas. En cada muestreo, las plantas se separaron en hoja, tallo, fruto (cápsula), cáliz y raíz, para obtener el peso de cada parte.

Cada unidad experimental estuvo compuesta de una planta establecida en contenedores de plástico flexible negro con capacidad de $14 \mathrm{~L}$, con orificios en la base para permitir un adecuado drenaje de la solución nutritiva, empleando como sustrato escoria volcánica basáltica roja, conocido comúnmente como tezontle rojo, con una granulometría de 3 a $7 \mathrm{~mm}$ de diámetro. La distancia entre macetas fue de $0.5 \mathrm{~m}$ y $1 \mathrm{~m}$ entre hileras.

Las fuentes de nutrimentos en la solución nutritiva, fueron los fertilizantes comerciales grado fertirriego: nitrato de potasio, nitrato de calcio, fosfato monopotásico, sulfato de potasio y sulfato de magnesio. Las concentraciones de micronutrimentos en $\mathrm{mg} \mathrm{L}^{-1}$ fueron: $\mathrm{Fe}, 3 ; \mathrm{Mn}, 1.48 ; \mathrm{Zn}, 0.24 ; \mathrm{Cu}, 0.12$; $\mathrm{B}, 0.28$; Mo 0.08 , con la adición de $0.04 \mathrm{~g} \mathrm{~L}^{-1}$ de la mezcla comercial Ultrasol ${ }^{\circledR}$ micro Mix. El pH de la solución nutritiva se ajustó con ácido sulfúrico a 5.5 \pm 0.2 , y se suministró con un sistema de riego por goteo con un volumen de $2.5 \mathrm{~L} \mathrm{~d}^{-1}$ los primeros 45 días después de transplante (ddt), posteriormente se suminstraron $5 \mathrm{~L} \mathrm{~d}^{-1}$ hasta los $105 \mathrm{ddt}$, procurando siempre el drenado de entre 10 y $15 \%$ de la solución nutritiva.

El agua de riego utilizada tiene la siguiente composición en meq $\mathrm{L}^{-1}$ : $\mathrm{Ca}^{+2}, 0.1 ; \mathrm{Mg}^{+2}, 0.028$; $\mathrm{K}^{+}, 0.19 ; \mathrm{Na}^{+}, 2.0$ y conductividad eléctrica de 0.44 dS $\mathrm{m}^{-1}$, RAS 7.1, pH 6.81 y CSR $1.8 \mathrm{meq} \mathrm{L}^{-1}$, que corresponde a una clasificación C2S1, de acuerdo a las normas Riverside. Posteriormente se procedió a realizar los ajustes correspondientes en las concentraciones iónicas de cada uno de los tratamientos de las soluciones nutritivas, considerando la composi- ción del agua.

El material vegetal se secó en estufa de secado a $60{ }^{\circ} \mathrm{C}$ en bolsas de papel estraza, por 72 $\mathrm{h}$ hasta peso constante. El peso fresco y peso seco de cada parte de la planta se obtuvo en una balanza digital compacta de precisión (Precisa BJ 2100D). En cosecha (105 ddt), los cálices se colectaron en cada una de las plantas, separando las cápsulas y obteniendo el peso total de cáliz cosechado. El secado de los cálices se realizó de manera tradicional mediante la exposición a los rayos del sol por cuatro días, hasta que perdieron $92 \%$ de humedad para luego determinar el peso seco de cálices por planta. A las muestras de biomasa seca aérea y raíz del tratamiento de potencial osmótico que tuvo la más alta producción de cáliz seco se les realizó el análisis químico de concentración de nutrimentos mediante los procedimientos estándares de laboratorio descritos por AlcántarGonzález y Sandoval-Villa (1999).

La extracción nutrimental acumulada por planta a través del ciclo de crecimiento del cultivo, se calculó por medio de la materia seca acumulada y su asignación en raíces, hojas, tallos, frutos y cálices, así como la concentración porcentual de $\mathrm{N}, \mathrm{P}, \mathrm{K}, \mathrm{Ca}$ y Mg. También se estimó el rendimiento de cálices secos, la extracción nutrimental acumulada y tasas de absorción nutrimental por hectárea, considerando una densidad de población de 20000 planta ha ${ }^{-1}$. Los datos obtenidos se sometieron a un análisis de varianza y prueba de comparación múltiple de Duncan $(\alpha>0.05)$ con el programa SAS.

\section{RESULTADOS}

El rendimiento de cáliz obtenido en los distintos tratamientos de $\psi_{o}$ en la solución nutritiva tuvo diferencias significativas. El menor rendimiento de cáliz $\left(0.88 \mathrm{t} \mathrm{ha}^{-1}\right)$ se obtuvo con un $\psi_{o}$ de -0.018 $\mathrm{MPa}$. En el intervalo -0.018 a $-0.072 \mathrm{MPa}$ (Figura 1), se observó una relación directa con el incremento del rendimiento, con la disminución del potencial osmótico; pero al disminuir el $\psi_{o}$ a $-0.090 \mathrm{MPa}$, el rendimiento fue menor $\left(1.56 \mathrm{t} \mathrm{ha}^{-1}\right)$. El tratamiento que tuvo el mayor rendimiento de cáliz seco con una media de $1.98 \mathrm{t} \mathrm{ha}^{-1}$, correspondió a la solución nutri- 
Tabla 1. Composición química de las soluciones nutritivas empleadas en los diferentes tratamientos.

\begin{tabular}{|c|c|c|c|c|c|c|c|}
\hline $\begin{array}{c}\text { Potencial osmótico } \\
\mathrm{MPa}\end{array}$ & $\mathrm{NO}_{3}^{-}$ & $\mathrm{H}_{2} \mathrm{PO}_{4}^{-}$ & $\begin{array}{l}\mathrm{SO}_{4}^{-2} \\
- \text { meq }\end{array}$ & $\begin{array}{c}\mathrm{K}^{+} \\
-1\end{array}$ & $\mathrm{Ca}^{2+}$ & $\mathrm{Mg}^{2+}$ & $\begin{array}{c}C E^{\dagger} \\
d S m^{-1}\end{array}$ \\
\hline-0.018 & 3 & 0.25 & 1.75 & 1.75 & 2.25 & 1.0 & 0.5 \\
\hline-0.036 & 6 & 0.50 & 3.5 & 3.50 & 4.50 & 2.0 & 1.0 \\
\hline-0.054 & 9 & 0.75 & 5.25 & 5.25 & 6.75 & 3.0 & 1.5 \\
\hline-0.072 & 12 & 1.00 & 7.0 & 7.00 & 9.00 & 4.0 & 2.0 \\
\hline-0.090 & 15 & 1.25 & 8.75 & 8.75 & 11.25 & 5.0 & 2.5 \\
\hline
\end{tabular}

${ }^{\dagger} \mathrm{CE}=$ Conductividad eléctrica, los valores tuvieron una variación de $\pm 0.2 \mathrm{dS} \mathrm{m} \mathrm{m}^{-1}$

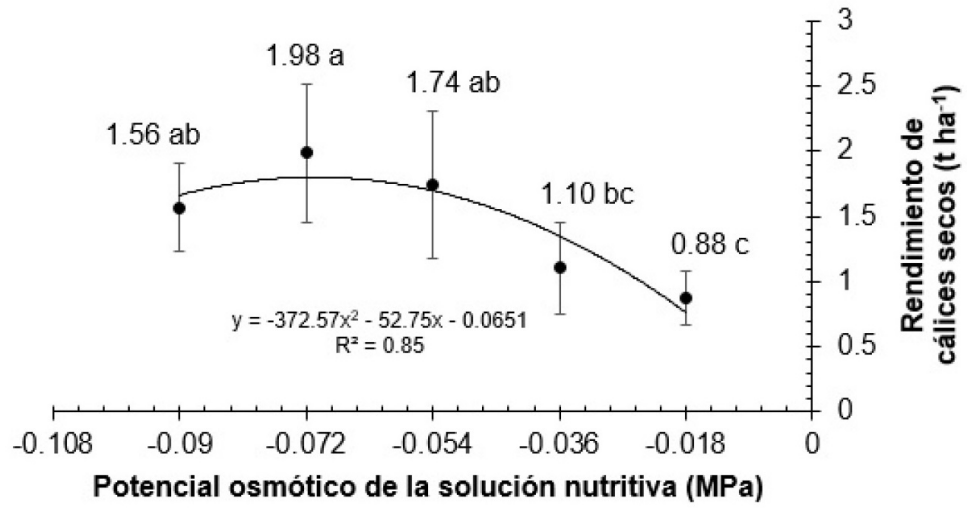

Figura 1. Rendimiento de cálices secos de jamaica (Hibiscus sabdariffa L.) variedad UAN-6 cultivada con soluciones nutritivas de diferente $\psi_{o}$. Medias con letras iguales no son estadísticamente diferentes (Duncan, $p>0.05$ ). Las barras representan las desviaciones estándar.

Tabla 2. Materia seca total acumulada por hectárea en los diferentes órganos de la planta de jamaica (Hibiscus sabdariffa L.) variedad UAN-6, al momento de la cosecha, empleando soluciones nutritivas con diferente potencial osmótico. Los valores se estimaron con una población de 20000 plantas ha $^{-1}$.

\begin{tabular}{|c|c|c|c|c|c|}
\hline \multirow[b]{2}{*}{$\begin{array}{c}\text { Potencial osmótico } \\
\mathrm{MPa}\end{array}$} & \multicolumn{5}{|c|}{ Partes de la planta } \\
\hline & Raíz & Hoja & $\begin{array}{c}\text { Tallo } \\
-\mathrm{kg} \mathrm{ha}^{-1}\end{array}$ & Fruto + cáliz & Total \\
\hline-0.018 & $231.37^{b}$ & $499.3^{b}$ & $1051.8^{a}$ & $2086.8^{b}$ & $3869.3^{b}$ \\
\hline-0.036 & $293.08^{a b}$ & $752.6^{a b}$ & $1285.3^{a}$ & $2570.2^{a b}$ & $4901.2^{a b}$ \\
\hline-0.054 & $347.52^{a}$ & $877.2^{a}$ & $1197.4^{a}$ & $3389.5^{a}$ & $5811.6^{a}$ \\
\hline-0.072 & $387.07^{a}$ & $1062.0^{a}$ & $1372.6^{a}$ & $3651.3^{a}$ & $6473.0^{a}$ \\
\hline-0.090 & $335.59^{a}$ & $848.3^{a}$ & $1265.7^{a}$ & $3162.6^{a b}$ & $5612.2^{a}$ \\
\hline
\end{tabular}

tiva con potencial osmótico de -0.072 MPa (Figura 1), aunque sin diferencias estadísticas con los tratamientos $-0.036,-0.054$ y $-0.090 \mathrm{MPa}$.

En la Tabla 2 se muestran los resultados obtenidos con la materia seca total acumulada (MST) y su asignación entre los órganos de la planta de jamaica. La acumulación de materia seca en la planta completa, tuvo una tendencia similar a lo obtenido con el rendimiento de cálices secos. La solución nutri- tiva con $\psi_{o}$ de $-0.018 \mathrm{MPa}$ tuvo la menor acumulación de MST en la planta de jamaica con $3869.3 \mathrm{~kg} \mathrm{ha}^{-1}$, en comparación con el tratamiento de $-0.072 \mathrm{MPa}$ con $6473.0 \mathrm{~kg} \mathrm{ha}^{-1}$ y la mayor producción de MST, aunque sin diferencias estadísticas con respecto a las soluciones con - $0.036,-0.054$ y $-0.090 \mathrm{MPa}$. Con excepción del tallo, en todos los demás órganos tuvieron diferencias significativas en la materia seca acumulada con los tratamientos de $\psi_{o}$ de -0.018 y 


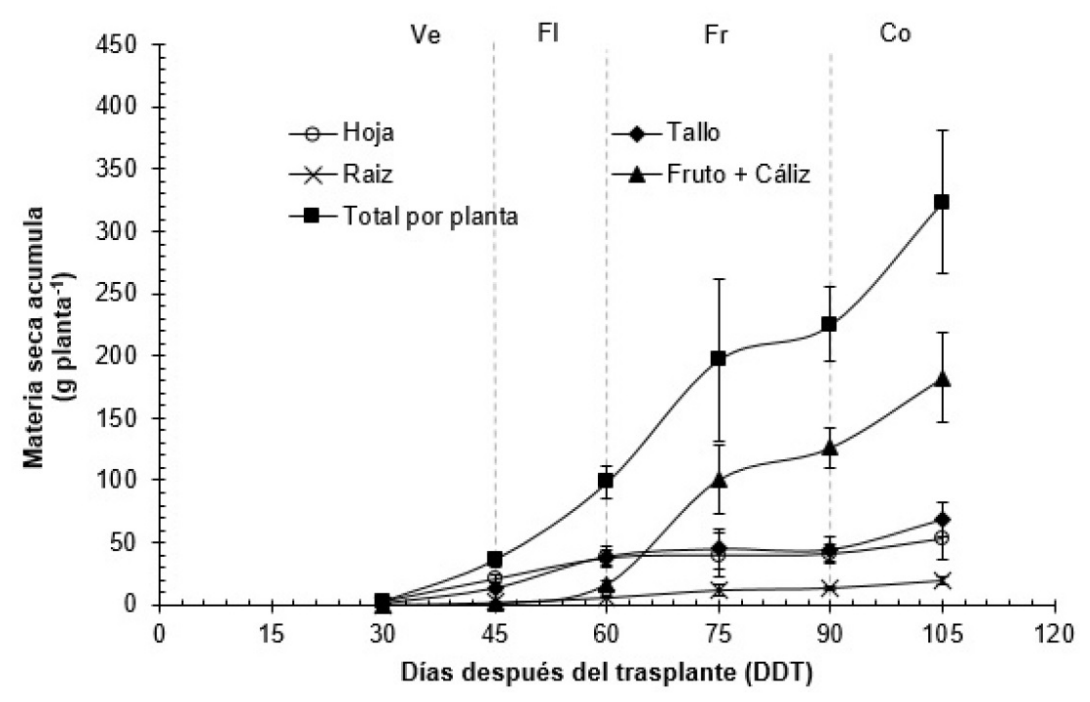

Figura 2. Materia seca acumulada en las diferentes partes de la planta de jamaica (Hibiscus sabdariffa L.) variedad UAN-6 durante las etapas fenológicas vegetativa, Ve; floración, Fl; fructificación, Fr; y cosecha, Co, cultivada con solución nutritiva de $\psi_{o}-0.072 \mathrm{MPa}$. Las barras representan el error estándar.

-0.036 MPa comparado con los tratamientos de 0.054, -0.072 y $-0.090 \mathrm{MPa}$ (Tabla 2). El fruto y cáliz fueron las partes de la planta que concentraron el $56.5 \%$ de la MST, seguido por el tallo con $21.24 \%$, hojas $16.25 \%$ y raíz $6 \%$. Se determinó que después de los $65 \mathrm{ddt}$, el fruto y cáliz es la parte de la planta que más contribuye al incremento de la MST (Figura 2).

La extracción de macronutrimentos en la biomasa total y en las diferentes partes que componen la planta de jamaica se presenta en la Tabla 3. El orden de extracción nutrimental en la biomasa total fue $\mathrm{N}>\mathrm{K}>\mathrm{Ca}>\mathrm{Mg}>\mathrm{P}$. El fruto junto con el cáliz son las partes de la planta que extrajeron mayor cantidad de $\mathrm{N}, \mathrm{P}, \mathrm{K}$ y $\mathrm{Mg}$, con valores que representan el $57,61,47$ y $57 \%$, respectivamente, de la extracción nutrimental de la biomasa total. La acumulación máxima de $\mathrm{P}, \mathrm{K}$ y $\mathrm{Mg}$ ocurrió en el fruto y cáliz, siguiendo en orden descendente tallo, hoja y raíz. En el caso del $\mathrm{Ca}$, su acumulación fue mayor en las hojas con un $51 \%$ del total, siguiéndole el fruto y cáliz con $33.7 \%$. Al comparar la extracción nutrimental de $\mathrm{P}$ en la biomasa total con respecto a Ca y $\mathrm{Mg}$, se aprecia que ambos nutrimentos se demandan en mayor cantidad que el $\mathrm{P}$ por el cultivo de jamaica, requiriéndose el Ca 3.4 veces más que el $P$.

Tabla 3. Extracción total de N, P, K, Ca y Mg en las diferentes partes de la planta de jamaica (Hibiscus sabdariffa L.) variedad UAN-6 al momento de la cosecha de cálices, obtenidos en el tratamiento de solución nutritiva con -0.072 MPa.

\begin{tabular}{|c|c|c|c|c|c|}
\hline Parte de la planta & $\mathrm{N}$ & $P$ & $\mathrm{~K}$ & $\mathrm{Ca}$ & $\mathrm{Mg}$ \\
\hline Raíz & $\overline{0.30^{d}}$ & $0.01^{d}$ & $0.07^{d}$ & $0.14^{C}$ & $0.06^{d}$ \\
\hline Hoja & $2.20^{c}$ & $0.16^{c d}$ & $1.55^{c d}$ & $2.82^{b}$ & $0.29^{c d}$ \\
\hline Tallo & $1.42^{c d}$ & $0.43^{c}$ & $3.08^{b c}$ & $0.70^{c}$ & $0.38^{c}$ \\
\hline Fruto + Cáliz & $5.31^{b}$ & $1.00^{b}$ & $4.31^{b}$ & $1.86^{b}$ & $1.00^{b}$ \\
\hline Total & $9.24^{a}$ & $1.62^{a}$ & $9.03^{a}$ & $5.52^{a}$ & $1.75^{a}$ \\
\hline
\end{tabular}

En la Figura 3, se aprecian las curvas de extracción nutrimental de N, P, K, Ca y Mg en la biomasa total durante las diferentes etapas fenológicas del cultivo de jamaica, se presentan tales resultados debido a que fue en el tratamiento en que se registró la mayor producción de cáliz seco. En los primeros 45 ddt de la etapa vegetativa, la tasa de acumulación de nutrimentos en la biomasa total fue menor comparado con las etapas posteriores (Tabla 4). Mientras que en el periodo de la antesis hasta la etapa de fructificación 

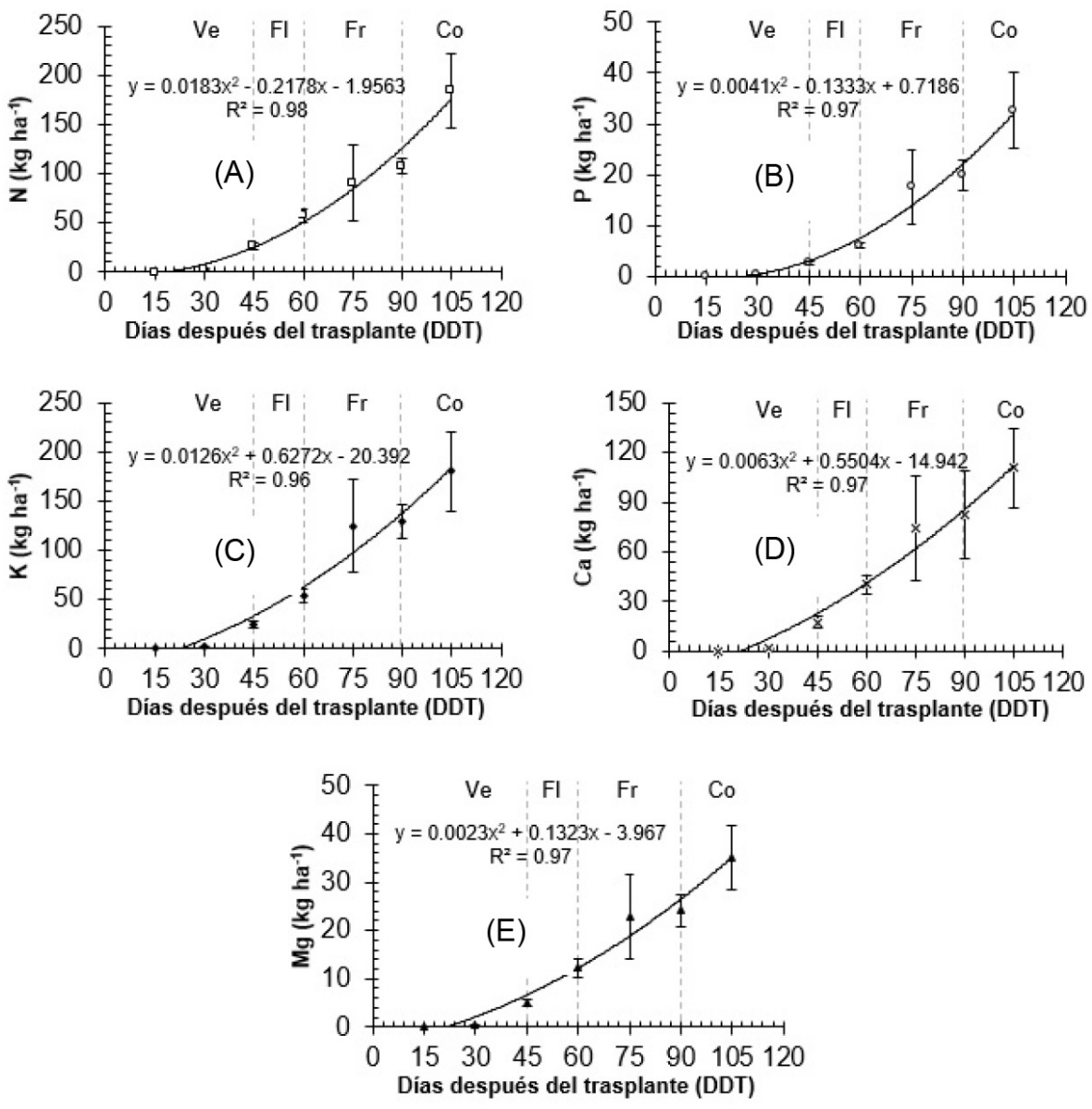

Figura 3. Curvas de extracción de macronutrimentos: A) Nitrógeno, B) Fósforo, C) Potasio, D) Calcio y E) Magnesio, durante las diferentes etapas fenológicas (vegetativa, Ve; floración, Fl; fructificación, Fr; y cosecha, Co) en el cultivo de jamaica (Hibiscus sabdariffa L.) variedad UAN-6. Los datos corresponden al tratamiento con $\psi_{o}$ de $-0.072 \mathrm{MPa}$. Las barras verticales representan el error estándar.

es donde se presentó un pronunciado incremento en la acumulación de nutrimentos y mayores tasas de absorción nutrimental. El potasio fue el nutrimento que se acumuló en mayor cantidad durante la etapa de fructificación. En el periodo de 75 a 90 ddt, disminuyó la tasa de absorción nutrimental y por consiguiente, se tuvo menor acumulación de nutrimentos en la biomasa total (Figura 2, Tabla 4). Después de los 90 ddt hasta la cosecha, se vuelve a presentar elevada acumulación de nutrimentos en la biomasa total, lo cual se explica porque durante este periodo ocurrió una nueva rebrotación vegetativa, que más tarde originó frutos y cálices. Este fenómeno puede
Tabla 4. Tasa de absorción de macronutrimentos durante el ciclo de vida del cultivo de jamaica (Hibiscus sabdariffa L.) variedad UAN-6, correspondiente al tratamiento de $-0.072 \mathrm{MPa}$

\begin{tabular}{cccccc}
\hline DDT $^{\dagger}$ & $\mathrm{N}$ & $\mathrm{P}$ & $\mathrm{K}$ & $\mathrm{Ca}$ & $\mathrm{Mg}$ \\
\cline { 5 - 6 } & & $\mathrm{kg} \mathrm{ha}^{-1}$ & $\mathrm{~d}^{-1}$ & & \\
\hline 15 & 0.009 & 0.001 & 0.006 & 0.006 & 0.002 \\
30 & 0.18 & 0.01 & 0.13 & 0.09 & 0.02 \\
45 & 1.58 & 0.17 & 1.56 & 1.06 & 0.30 \\
60 & 2.04 & 0.22 & 1.91 & 1.53 & 0.49 \\
75 & 2.25 & 0.77 & 4.72 & 2.24 & 0.71 \\
90 & 1.13 & 0.16 & 0.34 & 0.54 & 0.09 \\
105 & 5.14 & 0.85 & 3.39 & 1.91 & 0.73 \\
\hline
\end{tabular}

$\dagger$ DDT= Días después del transplante. 


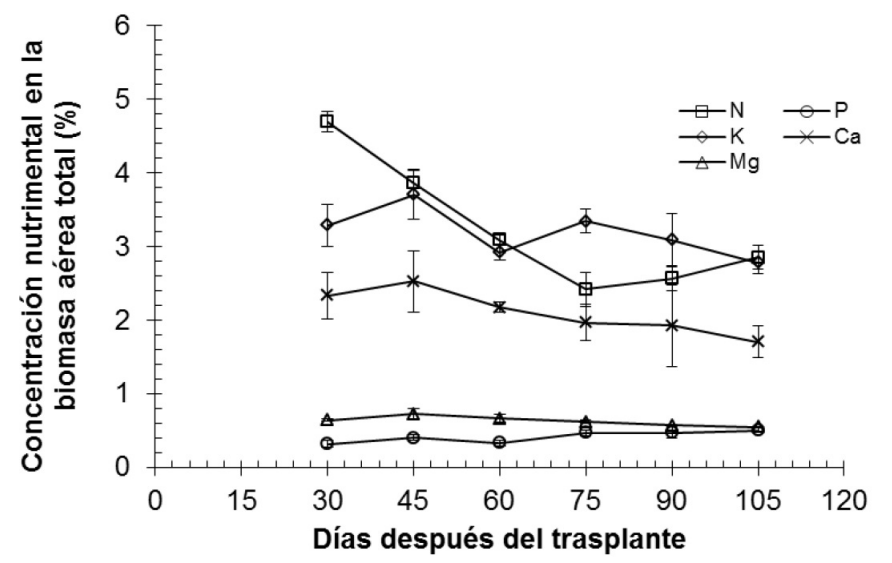

Figura 4. Concentración nutrimental en la biomasa total de jamaica (Hibiscus sabdariffa L.) variedad UAN-6, a través del ciclo de crecimiento de plantas, con solución nutritiva de $\psi_{o}$ de $-0.072 \mathrm{MPa}$.

tener impacto agronómico, en virtud de que mejorando la técnica de cosecha, es posible aprovechar comercialmente los cálices desarrollados; de lo contrario, muchos cálices de este último rebrote, se cosechan en estado inmaduro.

Durante el desarrollo del cultivo de jamaica, se presentaron dos etapas de mayor acumulación nutrimental, la primera entre los 61 y $75 \mathrm{ddt}$; y la segunda después de los 90 ddt hasta la cosecha, con valores máximos de absorción de nutrimentos de $5.14,0.85,3.39,1.91$ y $0.73 \mathrm{~kg} \mathrm{ha}^{-1} \mathrm{~d}^{-1}$ de $\mathrm{N}, \mathrm{P}$, $\mathrm{K}$, Ca y Mg, respectivamente (Tabla 4). La información generada acerca de la acumulación nutrimental y tasas de absorción de nutrimentos en el cultivo a través del tiempo, puede ser empleada en programas de fertirrigación y cultivos sin suelo.

La variación en la concentración nutrimental de $\mathrm{N}, \mathrm{P}, \mathrm{K}, \mathrm{Ca}$ y $\mathrm{Mg}$ en la biomasa total de las plantas cultivadas con la solución nutritiva de $-0.072 \mathrm{MPa}$ a través del tiempo, en las etapas fenológicas de jamaica se presenta en la Figura 4. En todos los casos se tuvo disminución en las concentraciones nutrimentales en el ciclo de cultivo. Los máximos valores durante el ciclo de crecimiento de las plantas de jamaica fueron para $\mathrm{N}$ y $\mathrm{K}$, seguido de $\mathrm{Ca}, \mathrm{Mg}$ y P. Durante la etapa vegetativa y floración, la concentración de $\mathrm{N}$ fue superior al resto de los macronutrimentos cuantificados; sin embargo, en la etapa de fructificación y cosecha, la concentración de $\mathrm{K}$ fue superior a los demás nutrimentos, incluido el $\mathrm{N}$, lo cual demuestra que las cápsulas de las plantas, acumulan mayor cantidad de este nutrimento, y coincide con el periodo de 75 a 90 ddt, donde se presentó la máxima absorción de K (Tabla 4).

En la Tabla 5 se presentan los valores del requerimiento interno nutrimental de $\mathrm{N}, \mathrm{P}, \mathrm{K}, \mathrm{Ca}$ y $\mathrm{Mg}$, expresado en \% de nutrimento en la biomasa total al momento de la cosecha, así como también en kg de nutrimento por tonelada de cáliz seco con contenido de humedad de $8 \%$. Los macronutrimentos que más requiere la jamaica es $\mathrm{N}$ y $\mathrm{K}$, mientras que el $\mathrm{P}$ y $\mathrm{Mg}$ son los que se requieren en menor cantidad.

Tabla 5. Requerimiento interno nutrimental de N, P, K, Ca y Mg en jamaica variedad UAN-6, expresado en \% del nutrimento en la biomasa total al momento de la cosecha y kg de nutrimento por tonelada de cáliz cosechado

\begin{tabular}{|c|c|c|}
\hline \multirow{2}{*}{ Nutrimento } & \multicolumn{2}{|c|}{ Requerimiento interno nutrimental ${ }^{\dagger}$} \\
\hline & $\%$ en la biomasa total & $\mathrm{kg} \cdot \mathrm{t}^{-1}$ de cáliz cosechado \\
\hline $\mathrm{N}$ & $2.86 \pm 0.17$ & $93.1 \pm 12.2$ \\
\hline$P$ & $0.50 \pm 0.03$ & $16.4 \pm 2.4$ \\
\hline $\mathrm{K}$ & $2.80 \pm 0.15$ & $91.0 \pm 12.2$ \\
\hline $\mathrm{Ca}$ & $1.71 \pm 0.22$ & $55.7 \pm 10.5$ \\
\hline $\mathrm{Mg}$ & $0.54 \pm 0.01$ & $17.7 \pm 2.3$ \\
\hline
\end{tabular}

${ }^{\dagger}$ Los valores \pm representan el error estándar.

\section{DISCUSIÓN}

Los tratamientos en potencial osmótico corres- 
ponden a CE que van de 1.0 a $2.5 \mathrm{dS} \mathrm{m}^{-1}$, por lo que los resultados evidencian que las plantas de jamaica pueden tolerar condiciones de hasta $2.5 \pm 0.2$ dS $\mathrm{m}^{-1}$ en la solución nutritiva, sin disminuir de forma significativa el rendimiento de cáliz seco. Un aspecto no considerado en esta investigación, fue la medición de la CE en el sustrato durante el ciclo del cultivo, pero es probable, que el nivel máximo de salinidad lo tenga el tratamiento con $2.5 \mathrm{dS} \mathrm{m}^{-1}$ como ocurre con frecuencia en los sistemas hidropónicos (Sonneveld y Voogt 2009). Por lo que valores de CE mayores a $2.5 \mathrm{dS} \mathrm{m}^{-1}$, pueden explorarse para conocer el umbral de tolerancia a la salinidad, así como determinar su efecto en la producción de metabolitos primarios y secundarios responsables del sabor, aroma, color y propiedades antioxidantes del cáliz de jamaica. En la jamaica, es posible tener un rendimiento máximo de cáliz seco superior a las $2 \mathrm{t} \mathrm{ha}^{-1}$, como lo reportan Basazinew y Bizuayehu (2017) y Nemati et al. (2018). Pero el rendimiento de cáliz seco es un parámetro muy sensible a las condiciones de cultivo, que debe tomarse en cuenta (Ottai et al. 2006).

El comportamiento en acumulación de materia seca permite inferir que los fotoasimilados de la planta, se concentran en las estructuras reproductivas, como lo reporta Atta et al. (2011) en nueve ecotipos de jamaica. El índice de cosecha (IC) en las plantas de jamaica cultivadas con diferentes tratamientos de potencial osmótico, varió de 0.25 a $0.30 \mathrm{~g} \mathrm{~g}^{-1}$. El menor valor correspondió al $\psi_{o}$ de $-0.018 \mathrm{MPa}$, lo cual pudo deberse al menor rendimiento de cálices secos. El mayor IC se obtuvo en los tratamientos de $\psi_{o}$ de -0.054 y $-0.072 \mathrm{MPa}\left(0.30 \mathrm{~g} \mathrm{~g}^{-1}\right)$, aunque sin diferencias estadísticas con respecto a los potenciales osmóticos de $-0.036\left(0.27 \mathrm{~g} \mathrm{~g}^{-1}\right)$ y $-0.090 \mathrm{MPa}(0.28 \mathrm{~g}$ $\left.\mathrm{g}^{-1}\right)$. El valor medio del IC, considerando los mejores tratamientos de potencial osmótico fue de $0.28 \mathrm{~g} \mathrm{~g}^{-1}$, mismo que se sugiere emplear para estimación de la biomasa seca total en el cultivo de jamaica, dado que considera una amplitud importante de la condición del potencial osmótico de la solución nutritiva. La alta extracción de $\mathrm{N}$ por el fruto con cáliz (5.1 $\mathrm{g}_{\text {planta }}{ }^{-1}$ ) puede explicarse porque el contenido de proteína en el cáliz y semillas es alto, con un valor de hasta 10.1 y $20 \%$ en base a peso seco, respectivamente (Fakir et al. 2012). La disminución en concentración nutrimental es típico en las especies vegetales, como ha sido descrito por Mills y Benton (2007).

Estos valores pueden emplearse para calcular la demanda nutrimental de acuerdo al rendimiento máximo alcanzable o rendimiento esperado en una condición agroecológica determinada, como ha sido propuesto por Rodríguez et al. (2001) y complementa la información agronómica obtenida hasta el momento (Caro-Velarde et al. 2012, Ruiz-González, Victorino-Ramírez 2014) para establecer programas de fertilización en el cultivo de jamaica. En este sentido, para calcular la dosis de fertilizante, solo se tendrá que evaluar el suministro edáfico nutrimental del sitio agrícola de interés y considerar la eficiencia de recuperación del fertilizante por el cultivo.

\section{CONCLUSIONES}

Los potenciales osmóticos de la solución nutritiva promovieron diferencias significativas en el rendimiento de cálices secos y acumulación de materia seca en plantas de jamaica. El rendimiento máximo de $1.98 \mathrm{t} \mathrm{ha}^{-1}$ de cálices secos se obtuvo con el tratamiento de $-0.072 \mathrm{MPa}$, y la mayor asignación de materia seca lo tuvo la parte del fruto y cáliz. La extracción nutrimental en la biomasa total fue en el orden $\mathrm{N}>\mathrm{K}>\mathrm{Ca}>\mathrm{Mg}>\mathrm{P}$. Los valores de requerimiento interno nutrimental obtenidos, permiten estimar la demanda nutrimental de $\mathrm{N}, \mathrm{P}, \mathrm{K}, \mathrm{Ca}$ y Mg para distintas metas de rendimiento de cálices secos de jamaica.

\section{AGRADECIMIENTOS}

Al proyecto SAGARPA-CONACYT No. 163972 "Validación de variedades de jamaica (Hibiscus sabdariffa L.) con alta concentración de bioactivos, alto rendimiento y tolerantes a enfermedades, determinación de plagas y enfermedades e innovación de la maquinaria agrícola para una producción sustentable" 


\section{LITERATURA CITADA}

Abo-Baker AA, Mostafa GG (2011) Effect of bio and chemical fertilizers on growth, sepals yield and chemical composition of Hibiscus sabdariffa at new reclaimed soil of South Valley Area. Asian Journal of Crop Science 3: $16-25$.

Alcántar-González G, Sandoval-Villa M (1999) Manual de análisis químico de tejido vegetal. Publicación especial No. 10. SMCS. Chapingo, México. 156p.

Ariza-Flores R, Serrano-Altamirano V, Navarro-Galindo S, Ovando-Cruz ME, Vásquez-García E, Barrios-Ayala A, et al. (2014) Variedades mexicanas de jamaica (Hibiscus sabdariffa L.) "alma blanca" y "rosalís" de color claro y "cotzaltzin" y "tecoanapa" de color rojo. Revista Fitotecnia Mexicana 37: 181-185.

Atta S, Hadiara HS, Yacoubou B, Benoît S, Issaka L, Mahamane S (2011) Yield character variability in Roselle (Hibiscus sabdariffa L.). African Journal of Agricultural Research 6: 1371-1377.

Babatunde FE, Mofoke ALE (2006) Performance of roselle (Hibiscus sabdariffa L.) as influenced by irrigation schedules. Pakistan Journal of Nutrition 5: 363-367.

Basazinew DG, Bizuayehu TA (2018) Effects of inter and intra row spacing on growth, yield and yield components of roselle (Hibiscus sabdariffa L.) at Wondo Genet, Southern Ethiopia. International Journal of Advanced Biological and Biomedical Research 6: 391-398.

Bugarín-Montoya R, Galvis-Spinola A, Sánchez-García P, García-Paredes D (2002). Demanda de potasio del tomate tipo saladette. Terra Latinoamericana 20: 391-399.

Bugarín-Montoya R, Virgen-Ponce M, Galvis-Spinola A, García-Paredes D, Hernández- Mendoza T, BojórquezSerrano I, et al. (2011) Extracción de nitrógeno en seis especies olerícolas durante su ciclo de crecimiento. Bioagro 23: 93-98.

Caro-Velarde FJ, Machuca-Sánchez ML, Flores-Berrios EP (2012) El cultivo de jamaica en Nayarit. Segunda edición. Universidad Autónoma de Nayarit. Nayarit, México. 103p.

Dahmardeh M (2012) Effect of mineral and organic fertilizers on the growth and calyx yield of roselle (Hibiscus sabdariffa L.). African Journal of Biotechnology 11: 10899-10902.

Egharevba RKA, Law-Ogbomo KE (2007) Comparative effects of two nitrogen sources on the growth and yield of roselle (Hibiscus sabdariffa) in the rainforest region: a case study of Benin-city, Edo State, Nigeria. Journal of agronomy 6: 142-146.

El-Naim AM, Ibrahim MI, Mohammed EAR, Elshiekh AI (2012) Evaluation of some local sorghum (Sorghum Bicolor L. Moench) Genotypes in Rain-Fed. International Journal of Plant Research 2: 15-20.

Etchevers BJD (1999) Técnicas de diagnóstico útiles en la medición de la fertilidad del suelo y el estado nutrimental de los cultivos. Terra Latinoamericana 17: 209-219.

Fakir MSA, Islam MM, Islam A, Islam F, Chowdhury MM (2012) Capsule growth and calyx protein content in Hibiscus sabdariffa L. var. sabdariffa. Journal of agroforestry and environment 6: 1-4.

Giginyu MB, Fagbayide JA (2009) Effect of nitrogen fertilizer on the growth and calyx yield of two cultivars of roselle in Northern Guinea Savanna. Middle-East Journal of Scientific Research 4: 66-71.

Greenwood JD, Cleaver TJ, Turner MK, Hunt J, Niendorf KB, Loquens SMH (1980) Comparison of the effects of potassium fertilizer on the yield, potassium content and quality of 22 different vegetable and agricultural crops. The Journal of Agricultural Science 95: 441-456. 
Hassan FAS (2009) Response of Hibiscus sabdariffa L. plant to some biofertilization treatments. Annals Agricultural Sciences 54: 437-446.

Mills HA, Benton JJr (2007) Plant analysis handbook II: A practical sampling, preparation, analysis, and interpretation guide. MicroMacro Publishing. USA. 422p.

Moreno-Pérez EC, Sánchez-del Castillo F, González-Molina L, Pérez-Mercado CA, Magaña-Lira N (2011) Efectos del volumen de sustrato y niveles de N-P-K en el crecimiento de plántulas de pepino. Terra Latinoamericana 29: 57-63.

Nemati M, Dahamardeh M, Khmmari E, Nejati M (2015) Effect of biofertilizer and manure application on economic yield and quality characteristics of roselle (Hibiscus Sabdariffa L.). Iranian Journal of Medicinal and Aromatic Plants 31: 610-625.

NICAEXPORT (2007) Estudio de inteligencia de mercados flor de Jamaica (Hibiscus sabdariffa L.) para México, Estados Unidos, Dinamarca y Costa Rica. Managua, Nicaragua. 111p.

Oyewole Cl, Mera M (2010) Response of roselle (Hibiscus sabdariffa L.) to rates of inorganic and farmyard fertilizers in the Sudan savanna ecological zone of Nigeria. Africal Journal of Agricultural Research 5: 23052309.

Ottai MES, Aboud KA, Mahmoud IM, El-Hariri DM (2006) Stability analysis of roselle cultivars (Hibiscus sabdariffa L.) under different nitrogen fertilizer environments. World Journal of Agricultural Sciences 2: 333-339.

Rodríguez SJ, Pinochet D, Matus F (2001) Fertilización de los cultivos. LOM Ediciones. Santiago de Chile. 117p.

Ruiz-González RO, Victorino-Ramírez L (2014) Respuesta del policultivo Jamaica-frijol-maíz a tres tratamientos de fertilización en Villaflores, Chiapas, México. Revista Ra Ximhai 10: 45-53.

SIAP (2017) Secretaría de Agricultura, Ganadería, Desarrollo Rural, Pesca y Alimentación (SAGARPA). Sistema de Información Agropecuaria. http://infosiap.siap.gob.mx/gobmx/datosAbiertos.php Fecha de consulta: 21 de julio de 2018.

Sonneveld C, Voogt W (2009) Plant nutrition of greenhouse crops. Springer. London, New York. 430p.

Steiner AA (1961) A universal method for preparing nutrient solutions of a certain desired composition. Plant and Soil 15: 134-154.

Vargas-Canales JM, Castillo-González AM, Pineda-Pineda J, Ramírez-Arias JA, Avitia-García E (2014) Extracción nutrimental de jitomate (Solanum lycopersicum L.) en mezclas de tezontle con aserrín nuevo y reciclado. Revista Chapingo Serie Horticultura 20: 71-88. 